\title{
Single-pixel polarimetric imaging
}

\author{
Vicente Durán, ${ }^{1,2, *}$ Pere Clemente,${ }^{1,3}$ Mercedes Fernández-Alonso, ${ }^{1,2}$ Enrique Tajahuerce,,${ }^{1,2}$ and Jesús Lancis ${ }^{1,2}$ \\ ${ }^{1}$ Institut de Noves Tecnologies de la Imatge (INIT), Universitat Jaume I, E12080 Castelló, Spain \\ ${ }^{2}$ GROC•UJI, Departament de Física, Universitat Jaume I, E12071 Castelló, Spain \\ ${ }^{3}$ Servei Central d'Instrumentació Científica, Universitat Jaume I, E12080 Castelló, Spain \\ *Corresponding author: vduran@sg.uji.es
}

Received November 18, 2011; revised January 16, 2012; accepted January 16, 2012; posted January 17, 2012 (Doc. ID 158463); published February 22, 2012

\begin{abstract}
We present an optical system that performs Stokes polarimetric imaging with a single-pixel detector. This fact is possible by applying the theory of compressive sampling to the data acquired by a commercial polarimeter without spatial resolution. The measurement process is governed by a spatial light modulator, which sequentially generates a set of preprogrammed light intensity patterns. Experimental results are presented and discussed for an object that provides an inhomogeneous polarization distribution. (c) 2012 Optical Society of America

OCIS codes: $120.5410,110.5405,230.6120$.
\end{abstract}

The aim of polarimetric imaging (PI) is to measure spatially resolved polarization properties of a light field, an object, or an optical system [1]. The measured quantities are usually the Stokes parameters of light or the Mueller matrix that characterizes a sample or a system. The use of PI has spread into a great variety of optical applications, including scene analysis and target detection [2], polarization-sensitive microscopy [3] , and segmentation of rough surfaces [4]. In the field of biomedical imaging, polarimetric techniques have been used for enhanced visualization of biological samples at different depths [5] as well as in vivo detection and diagnosis of cancerous tumors in tissues [6,7]. PI can be also combined with optical coherence tomography [8], ophthalmic adaptive optics [9], and hyperspectral imaging $[\underline{10}, 11]$.

In a different research avenue, compressive sampling (CS) has emerged as a novel sensing paradigm to perform image measurement in a compressed digital form [12]. More precisely, CS states that an $N$-pixel image of an object can be reconstructed from $M<N$ linear measurements with a high degree of fidelity. This is possible by exploiting the "sparsity" of natural images, that is, the fact that they can be expressed in a conventional function basis and only a few terms are nonzero. If a random subset of the expansion coefficients is experimentally determined (e.g., by an iterative acquisition process), CS theory ensures that the object can be recovered by solving a convex optimization program. One of the most outstanding applications of CS is the design of a singlepixel camera [13], which has promising applications at spectral regions where image sensors are impractical or inexistent [14].

In this Letter, we extend the concept of single-pixel imaging by CS to the design of a passive PI camera. As a proof of concept, we present a PI technique to measure spatially resolved Stokes parameters using a widespread commercial polarimeter, as those designed for free-space and fiber-based measurements, which provides the state of polarization (SOP) of an optical beam as a whole, that is, without spatial resolution. The high dynamic range (up to $70 \mathrm{~dB}$ ), broad wavelength range, and accuracy on the Poincaré sphere of such commercial single-pixel polarimeters are readily transferred to PI. This fact opens a wide spectrum of application possibilities and eliminates the demand of sophisticated methods to optimize the design of current expensive polarimetric cameras based on pixelated sensors $[\underline{7}, \underline{15}]$. The CS algorithm is at the heart of our optical design, where the time-multiplexed acquisition process is brought off by a programmable spatial light modulator (SLM).

The operation principle of a commercial Stokes polarimeter (SP) is the measurement of the irradiance of a light field whose SOP is modulated by a polarization state analyzer (PSA). In our SP, sketched in Fig. 1, the PSA is formed by two voltage-controlled liquid-crystal variable retarders $\left(\mathrm{LCVR}_{1}\right.$ and $\left.\mathrm{LCVR}_{2}\right)$ and a polarizing beam splitter (PBS). Two photodiodes $\left(\mathrm{PD}_{1}\right.$ and $\left.\mathrm{PD}_{2}\right)$ are respectively located at the output ports of the PBS. Despite of slight (and measurable) losses, the sum of the signals of $\mathrm{PD}_{1}$ and $\mathrm{PD}_{2}$ gives the total irradiance $I_{0}$ impinging onto the SP. The SOP of the input light, given by the Stokes vector $\left(I_{0}, S_{1}, S_{2}, S_{3}\right)^{T}$, can be obtained with the aid of the Stokes-Mueller formalism. When the retardances of $\mathrm{LCVR}_{1}$ and $\mathrm{LCVR}_{2}$ are $\delta_{1}$ and $\delta_{2}$, respectively, the irradiance $I_{\mathrm{PD}}$ measured by one photodiode is given by

$$
I_{\mathrm{PD}}\left(\delta_{1}, \delta_{2}\right)=m_{00}\left(\delta_{1}, \delta_{2}\right) I_{0}+\sum_{i=1}^{3} m_{0 i}\left(\delta_{1}, \delta_{2}\right) S_{i},
$$

where $m_{0 k}(k=0, \ldots, 3)$ are the voltage-dependent elements of the first row of the PSA Mueller matrix. These elements are determined through a proper calibration

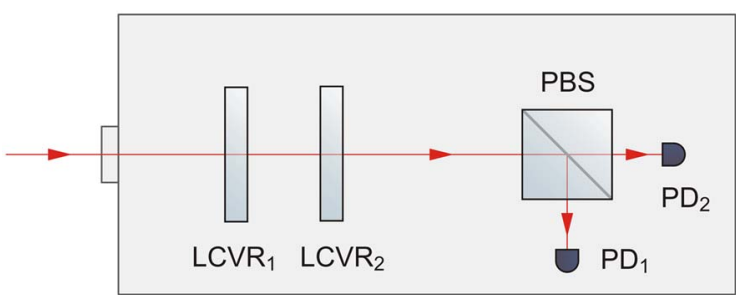

Fig. 1. (Color online) Scheme of the SP used as detector. 
process, out of the scope of the present Letter, and usually performed by the manufacturer. The SOP of the incoming light is derived by reconfiguring the PSA and measuring (at least) three values of $I_{\mathrm{PD}}$, together with the irradiance $I_{0}$. In practice, the LCVRs perform a retardance sweep, and the input SOP is obtained through a least-square fitting routine to minimize measurement errors [16]. As the retardance of the LCVRs strongly depends on the light frequency, the device must be recalibrated if the input wavelength is changed. The quantities displayed by the SP are usually the normalized version of the Stokes parameters, $\sigma_{i}=S_{i} / I_{0}(i=1, \ldots, 3)$.

The foundations of single-pixel imaging by CS can be summarized as follows. Consider an unknown object whose image (with a preestablished resolution of $N$ pixels) is rearranged in an $N \times 1$ column $x$. Suppose that this image is sparse (compressible) when expressed in terms of a conventional basis of functions, $\Psi=\left\{\psi_{l}\right\}$ ( $l=$ $1, \ldots, N)$. Mathematically, $x=\Psi s$, where $\Psi$ is a $N \times N$ matrix that has the vectors $\left\{\psi_{l}\right\}$ as columns and $s$ is the $N \times 1$ vector composed of the expansion coefficients. Only a small collection of these coefficients is nonzero. In order to determine $x$, a detector without spatial resolution measures the projections of the object image on a basis of $M$ intensity patterns $\phi_{m}(m=1, \ldots, M)$ of $N$-pixel resolution. This measurement process can be written as

$$
y=\Phi x=\Phi(\Psi s)=\Theta s,
$$

where $y$ is the $M \times 1$ column formed by the measured projections and $\Phi$ is the $M \times N$ sensing matrix. Each row of $\Phi$ is an intensity pattern $\varphi_{m}$, and the product of $\Phi$ and $\Psi$ gives the $M \times N$ matrix $\Theta$ acting on $s$. CS theory asserts that $x$ can be retrieved with high probability from a random subset of coefficients $(M<N)$ in the $\Psi$ domain. As the above equation represents an underdetermined matrix relation, a reconstruction algorithm is required. This step is performed by minimizing the $l_{1}$ norm of $s$ subjected to the restriction given by Eq. (2). If the measurements $\left\{y_{m}\right\}$ are affected by noise, the restriction of the CS algorithm is relaxed and the problem is reformulated with inequality constraints $[\underline{12}, 13]$.

A single-pixel CS camera basically consists of an SLM, which generates the intensity patterns $\left\{\phi_{m}\right\}$, and a light detector (without spatial resolution), which captures the irradiances $\left\{y_{m}\right\}$. A suitable SLM for CS imaging, usually found in video projection systems, is a display formed by voltage-controlled liquid-crystal (LC) cells [17]. Another option is a digital micromirrror device (DMD), composed of an array of micromirrors that can rotate between two positions so only selected portions of the incoming light beam are reflected in a given direction [13]. An example of a detector for a single-pixel camera is a photodiode, which measures the total irradiance of the light coming from an object for each pattern generated by the SLM.

The above optical architecture can be adapted to perform PI by simply using an SP as detector. The linearity of Eq. (1) implies that each Stokes parameter $S_{i}^{\mathrm{SP}}$ provided by the SP is an integrated quantity, that is, the sum of the values taken by $S_{i}$ at each point of the input light beam. Therefore, the measurement process expressed by Eq. (2) can be separately applied to each Stokes param-

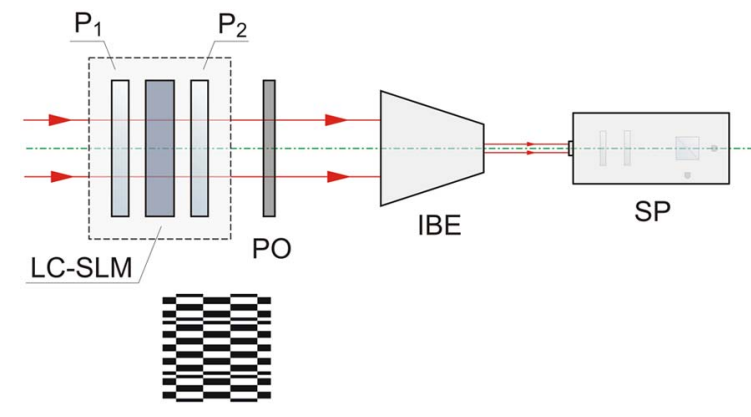

Fig. 2. (Color online) Setup for single-pixel imaging polarimetry. An example of a binary intensity pattern displayed by the SLM is also shown.

eter, whose spatial distribution (now described by an $N$ pixel matrix) is recovered using $M<N$ polarimetric measurements. In this way, the problem of measuring a spatial-dependent Stokes vector is equivalent to resolve three times the CS algorithm of single-pixel imaging. A general scheme of the proposed PI system is depicted in Fig. 2. A collimated (unpolarized) laser beam passes through an LC SLM, which generates a preprogrammed set of intensity patterns. Next, the light impinges onto a polarization object (PO), placed just after the modulator to produce a space-variant Stokes vector. As an LC SLM is a polarization-dependent device, it must be sandwiched between properly oriented linear polarizers $\left(P_{1}\right.$ and $P_{2}$ ). Therefore, the object is illuminated with linearly polarized light. After emerging from the object, the light is guided to the SP by means of an afocal element [like an inverted beam expander (IBE)]. Such an element fits the beam width to the typically small entrance window of the SP. This coupling optics is essential to ensure that all the light emerging from the object is collected by the $\mathrm{SP}$ and to preserve the normal incidence, which contributes to the optimal performance of the polarimeter.

The light source used in our experiment was an $\mathrm{He}-\mathrm{Ne}$ laser emitting at $0.6328 \mu \mathrm{m}$. The LC SLM was a transmissive twisted nematic LCD (TNLCD) with SVGA resolution $(800 \times 600$ pixels $)$ and a pixel pitch of $32 \mu \mathrm{m}$. To reach an intensity modulation regime, the LC SLM was sandwiched between two linear polarizers, respectively oriented parallel and normal to the input molecular director of the TNLCD, which was previously determined by a polarimetric technique [18]. Pixels were individually addressed by sending gray-level images to the TNLCD. Each gray level corresponded to a transmitted intensity level, ranging from total extinction (dark state) to maximum transmission (bright state). We chose the WalshHadamard functions as the reconstruction basis $\Psi$. This election was particularly useful because the intensity patterns $\left\{\varphi_{m}\right\}$ generated by the TNLCD were binary objects (see Fig. 2). The corresponding images addressed to the LC SLM had a resolution of $64 \times 64$ cells. The area of each cell was $64 \mu \mathrm{m} \times 64 \mu \mathrm{m}$. The TNLCD sequentially projected over the object a set of 1225 preprogrammed binary patterns ( $\sim 30 \%$ of the Nyquist criterion). The SP was synchronized with the modulator by means of custom software written with LabVIEW. For each realization, we measured the values of the Stokes parameters $\left\{S_{i}^{\mathrm{SP}}\right\}(i=1, \ldots, 3)$ as well as the signals of $\mathrm{PD}_{1}$ and $\mathrm{PD}_{2}$. The PI system worked at the maximum measurement 


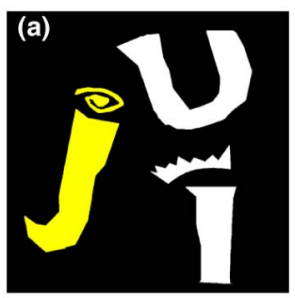

Stokes parameter $\sigma$

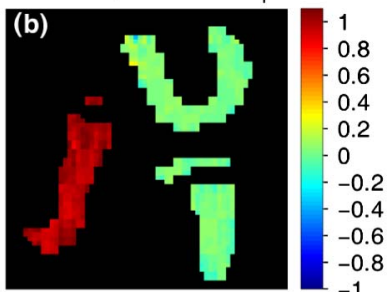

Stokes parameter $\sigma_{2}$

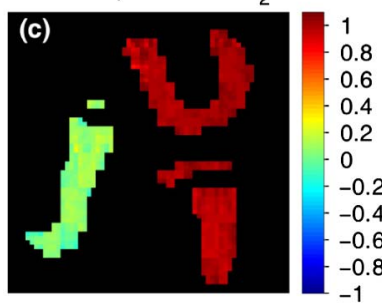

Stokes parameter $\sigma_{3}$

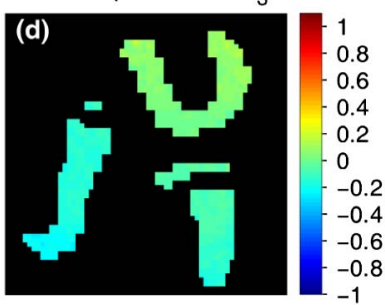
Fig. 3. (Color online) (a) High-resolution image $(1024 \times 1024$ pixels $)$ of the object used in the experiment, which consists of an amplitude mask with a cellophane film covering the zone colored in yellow. Pseudocolor images (of $64 \times 64$ pixels) for the Stokes parameters (b) $\sigma_{1}$, (c) $\sigma_{2}$, and (d) $\sigma_{3}$.

rate of the SP (10 Stokes vectors per second). This was the speed limiting factor, since the refreshing frequency of the display was $60 \mathrm{~Hz}$.

The selected object, shown in Fig. 3(a), was a cellophane film, acting as linear retarder, attached to an amplitude mask-our university logotype, UJI. Linearly polarized light emerging from the polarizer $\mathrm{P}_{2}$ illuminated the object. By covering only the J capital letter with the cellophane, an inhomogeneous polarization distribution was generated since the polarization of the light coming from this letter was approximately rotated by the cellophane film. The parameters of the polarization ellipse (the azimuth $\alpha$ and the ellipticity $e$ ) of the light passing through the $\mathrm{J}$ were measured by the SP (blocking the light emerging from the other part of the object). For this measurement, the TNLCD was configured in its bright state. The results were $\alpha_{J}=8.62^{\circ}$ and $e_{J}=-0.07$. Repeating the process for the $\mathrm{U}$ and I letters, the measured parameters were $\alpha_{U, I}=42.22^{\circ}$ and $\alpha_{U, I}=0.003$.

The programming code used in the CS reconstruction was the so-called l1eq-pd, which solves the standard basis pursuit problem using a primal-dual algorithm [19]. Pseudocolor plots for the normalized Stokes parameters are shown in Figs. 3(b) $-3(d)$, respectively. These images reveal an apparent uniformity within the different parts of the object. The spatial distributions for $\alpha$ and $e$ were calculated from the Stokes parameters through conventional expressions (see, for example, [20]). The mean values of the ellipse parameters for each part of the object were $\quad\left(\left\langle\alpha_{J}\right\rangle=2.46^{\circ} \pm 1.4^{\circ}, \quad\left\langle e_{J}\right\rangle=-0.08 \pm 0.02\right) \quad$ and $\left(\left\langle\alpha_{U, I}\right\rangle=43.61^{\circ} \pm 1.1^{\circ},\left\langle e_{U, I}\right\rangle=-0.01 \pm 0.04\right)$. The assigned uncertainties were the standard deviations of each distribution. The above results were in good agreement with the values previously measured by the SP. The major discrepancy was found for $\alpha_{J}\left(\sim 6^{\circ}\right)$, which only represents $\sim 3 \%$ of the total range of values (from $-90^{\circ}$ to $90^{\circ}$ ) that can be taken by the azimuth.

In conclusion, we have demonstrated the possibility of performing spatially resolved Stokes polarimetry via CS. As a proof of concept, we have proposed a system that allows us to convert a commercial nonpixelated SP into a polarimetric imager. Although we have focused on an SP based on LC technology, our conclusions are valid for other types of polarimeters, provided that the selected device is itself spatially homogeneous and the relationship between the measured signals and the Stokes parameters is linear, as in Eq. (1). Concerning the SLM that controls the data acquisition process, a TNLCD has been used to project over an object the intensity patterns required by CS. Another arrangement can be assayed by using an SLM insensitive to polarization, like a DMD, since in that case the object can be placed before the SLM. The combination of DMDs with fast SPs may lead to the design of a PI system working at very high frequencies $(\sim 1 \mathrm{KHz})$, which opens the door to near-real-time applications.

This work was supported by the Spanish Ministerio de Ciencia e Innovación (MICINN) grants and FIS201015746.

\section{References}

1. J. E. Solomon, Appl. Opt. 20, 1537 (1981).

2. J. S. Tyo, D. L. Goldstein, D. B. Chenault, and J. A. Shaw, Appl. Opt. 45, 5453 (2006).

3. R. Oldenbourg, Nature 381, 811 (1996).

4. P. Terrier, V. Devlaminck, and J. M. Charbois, J. Opt. Soc. Am. A 25, 423 (2008).

5. S. G. Demos and R. R. Alfano, Appl. Opt. 36, 150 (1997).

6. J. S. Baba, J. R. Chung, A. H. DeLaughter, B. D. Cameron, and G. L. Cote, J. Biomed. Opt. 7, 341 (2002).

7. B. Laude-Boulesteix, A. De Martino, B. Drévillon, and L. Schwartz, Appl. Opt. 43, 2824 (2004).

8. J. F. de Boer and T. E. Milner, J. Biomed. Opt. 7, 359 (2002).

9. H. Song, Y. Zhao, X. Qi, Y. Toco Chui, and S. A. Burns, Opt. Lett. 33, 137 (2008).

10. D. A. Glenar, J. J. Hillman, B. Saif, and J. Bergstralh, Appl. Opt. 33, 7412 (1994).

11. K. Oka and T. Kato, Opt. Lett. 24, 1475 (1999).

12. E. J. Candès and M. B. Wakin, IEEE Signal Process. Mag. 25, 21 (2008).

13. M. F. Duarte, M. A. Davenport, D. Takhar, J. N. Laska, T. Sun, K. F. Kelly, and R. G. Baraniuk, IEEE Signal Process. Mag. 25(2), 83 (2008).

14. W. L. Chan, K. Charan, D. Takhar, K. F. Kelly, R. G. Baraniuk, and D. M. Mittleman, Appl. Phys. Lett. 93, 121105 (2008).

15. D. S. Sabatke, M. R. Descour, E. L. Dereniak, W. C. Sweatt, S. A. Kemme, and G. S. Phipps, Opt. Lett. 25, 802 (2000).

16. S. R. Davis, R. J. Uberna, and R. A. Herke, "Retardance sweep polarimeter and method," U.S. patent 6,744,509 (June 1, 2004).

17. F. Magalhaes, F. M. Araujo, M. V. Correira, M. Abolbashari, and F. Farahi, Appl. Opt. 50, 405 (2011).

18. V. Durán, J. Lancis, E. Tajahuerce, and Z. Jaroszewicz, J. Appl. Phys. 97, 043101 (2005).

19. http: \www.l1-magic.org.

20. C. Brosseau, Fundamentals of Polarized Light, 1st ed. (Wiley, 1998). 